\title{
Energetics and Population of Molecules at Microscopic Liquid and Solid Surfaces
}

\author{
Hongfei Wang, ${ }^{\dagger}$ Elsa C. Y. Yan, Yan Liu, and Kenneth B. Eisenthal* \\ Department of Chemistry, Columbia University, New York, New York 10027
}

Received: December 11, 1997; In Final Form: March 26, 1998

\begin{abstract}
A new application of second harmonic generation to selectively probe the surface of microscopic size centrosymmetric structures in centrosymmetric bulk media has been applied to polymer beads in aqueous solution and to oil droplets in an oil/water emulsion. The free energies of adsorption and the surface densities of organic molecules adsorbed from aqueous solution to $1 \mu \mathrm{m}$ polystyrene bead surfaces and to $230 \mathrm{~nm}$ tetradecane oil droplet surfaces have been determined. The potential use of this method as a chemical and biochemical sensor without the need for a fluorescent or magnetic tag is noted.
\end{abstract}

\section{Introduction}

The interface selectivity of second harmonic generation (SHG) and sum frequency generation (SFG) have led to their emergence in recent years as powerful spectroscopic probes of equilibrium and dynamic phenomena at interfaces. Among the interfaces studied with these methods are liquid/gas, liquid/ liquid, liquid/solid, solid/gas, and solid/solid interfaces. ${ }^{1-5}$ The origin of the interface specificity of these second-order nonlinear spectroscopies is the intrinsically noncentrosymmetric structure of interfaces. It is commonly assumed that SHG and SFG are electric dipole forbidden in centrosymmetric systems but not at the noncentrosymmetric interface. ${ }^{6,7}$ A consequence of this viewpoint is that most SHG and SFG studies have been carried out on noncentrosymmetric planar surfaces that separate centrosymmetric bulk media, e.g., bulk liquids, gases, and centrosymmetric solids. There is no strong coherent SHG or SFG radiated from bulk centrosymmetric systems because of symmetry reasons. The symmetry argument is that for every molecule oriented in one direction there is one oriented in the opposite direction. The nonlinear polarizations induced in these oppositely oriented molecules are of opposite phase with respect to each other and thus cancel. Therefore their net second-order polarization is zero, and there is no coherent SHG or SFG radiated from the bulk. If it was otherwise, the bulk signal would have overwhelmed the interface signal because of the much greater amount of material in the bulk than at the interface.

Although coherent SHG and SFG is not generated in bulk centrosymmetric media owing to the cancellation discussed above, the fluctuations in molecular density and molecular orientation in bulk solution can disrupt the phase cancellation. In this way incoherent second-order light scattering, called hyper-Rayleigh scattering, is generated and has been observed in bulk centrosymmetric media. ${ }^{8-9}$ SHG can also be generated by individual large noncentrosymmetric entities in bulk solution, as has been observed in randomly oriented suspensions in solution. ${ }^{10}$ The origin of the SHG signal in this case was chiefly the noncentrosymmetric interior region of the individual microscopic particles but not the surfaces of the microscopic particles. Semiconductor quantum dots and metallic crystals

\footnotetext{
* To whom correspondence should be addressed.

Present address: Department of Chemistry, University of Pennsylvania, Philadelphia, PA 19104, and Dupont Marshall Laboratory, Philadelphia, PA 19146.
}

also give rise to SHG, which is also attributed to their noncentrosymmetric structure. ${ }^{11}$

At this point we want to look more closely at the relation between the symmetry of the system and the generation of light at $2 \omega$ and at the sum frequency $\omega_{1}+\omega_{2}$ of the incident light fields. Although SHG and SFG are commonly asserted to be electric dipole forbidden in centrosymmetric systems, this is true only if the system is centrosymmetric on length scales much less than the coherence length of the process. For micron size centrosymmetric particles, the local surface symmetry is not centrosymmetric. Since the "opposite" surfaces of the particle are separated by distances of the order of the coherence length of the proces and are not locally centrosymmetric, there is no phase cancellation, and their second-order polarizations do not cancel. In addition, the amplitude of the incident light field can differ at different positions on the surface of micron size particles. Thus the induced second-order polarizations from "opposite" positions on the surface would have different amplitudes and would not cancel. Recent experiments support this noncancellation, and strong SH signals from polystyrene sulfate particles of microscopic size have been reported. ${ }^{12}$ Among the microscopic centrosymmetric particles, for which we have observed strong SH signals, are polymer beads, charged polymer beads, oil droplet/water interfaces in emulsions, liposomes, clay particles, and semiconductor colloids. A strong resonant sum frequency signal at $\omega_{1}+\omega_{2}(278 \mathrm{~nm})$ has been observed for polystyrene beads in aqueous solution. ${ }^{14}$ The sizes of the microstructures so far studied are in the range of 0.06 $\mu \mathrm{m}$ to several microns. On the basis of these recent findings, we note that the application of SHG and SFG to these microscopic systems provides a powerful new approach in the investigation of the physical, chemical, and biological properties of microscopic particle interfaces. ${ }^{13}$

In this paper we report on the use of SHG to measure the population of organic molecules adsorbed to microparticle interfaces. Using a Langmuir model, modified to include the depletion of the bulk solute population due to surface adsorption, we obtain the adsorption free energy, which is the thermodynamic quantity that determines the interface population of the adsorbed species. These SHG measurements also yield the value of the maximum density of adsorbates at the microparticle surface. Unlike traditional adsorption methods, the SHG technique is a nonperturbing method for measuring adsorption 


\section{SCHEME 1}
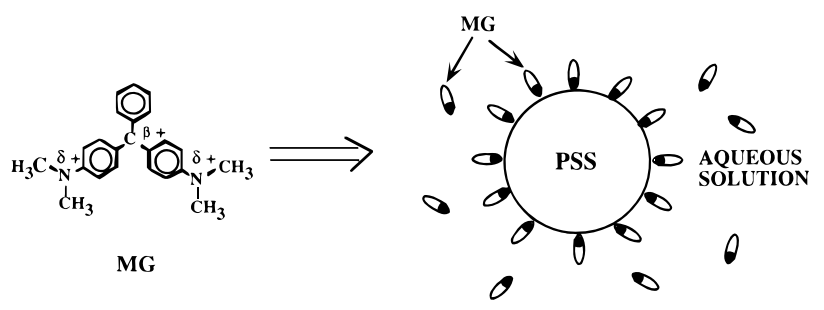

of solute molecules from a bulk solution to the surface of solid particles suspended in the solution. In one of the traditional methods centrifugation is employed. ${ }^{15-22}$ Chemical analysis of the supernatant solution is used to determine the number of solute molecules that have been adsorbed from the bulk solution. In this method it is difficult to establish that solute molecules, and water molecules in which they are immersed, have not been trapped in the centrifugation process. The results are therefore subject to errors. It is furthermore to be noted that the centrifugation method is not a surface-specific method, in contrast to the SHG method, which is surface-specific. Nonetheless the centrifugation method is of considerable value as a qualitative adsorption method because of its simplicity and because of the very wide class of solid particles that can be studied. It should be observed that neither centrifugation nor other traditional methods can be used to measure the adsorption of molecules to the liquid/liquid interface of emulsions, whereas the SHG method can be used as we shall describe below.

The experiments reported here were carried out on two different types of microscopic structures. One system is polystyrene sulfate (PSS) microspheres in aqueous solution, and the other is a $n$-tetradecane-water emulsion consisting of $n$-tetradecane (oil) droplets in a water solution. The organic molecule malachite green $(\mathrm{MG})$ was selected as the interface adsorbing species, in part because it is two-photon resonant with the incident light and consequently has a large second-order nonlinearity. Its adsorption to the PSS surface is schematically indicated below in Scheme 1.

The amplitude of the second harmonic signal was measured as a function of the malachite green concentration in the bulk liquid. From these measurements of the malachite green adsorption, we obtain the adsorption free energy, which is the reversible work to bring a malachite green molecule from the bulk region to the interface. It is this quantity that determines the distribution of malachite green molecules between the bulk and interface locations. From these same measurements we also obtain the maximum number of molecules, in this case malachite green, that can be adsorbed to the microparticle interface. It is to be noted that the measurement of the adsorption isotherm to the oil/water emulsion interface reported here is the first direct measurement of adsorption to an emulsion interface.

\section{Experimental Section}

The setup for the second harmonic measurements is shown in Figure 1. An $82 \mathrm{MHz}$ repetition rate Ti:sapphire oscillator producing $100 \mathrm{fs}$ pulses at $854 \mathrm{~nm}$ were used for the PSS experiments, and pulses at $846 \mathrm{~nm}$ were used for the emulsion system. The fundamental light is focused into a $0.2 \mathrm{~cm}$ sample cell. Second harmonic photons are detected in the transmitted direction using single photon counting methods. Filters and a monochromator separate the second harmonic photons from the fundamental and any background signals.

The solid particles used in these experiments were $1.05 \pm$ $0.03 \mu \mathrm{m}$ diameter monodisperse polystyrene sulfate (PSS)

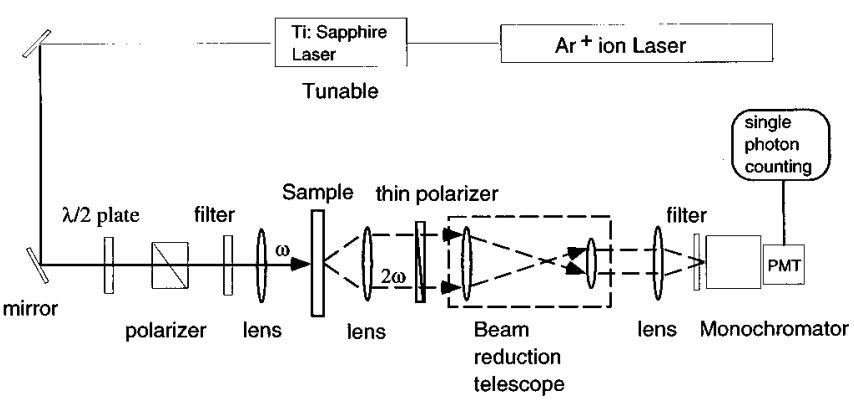

Figure 1. Experimental setup for SHG measurement of the density of molecules adsorbed to the surface of microscopic particles and microscopic structures in bulk solution.

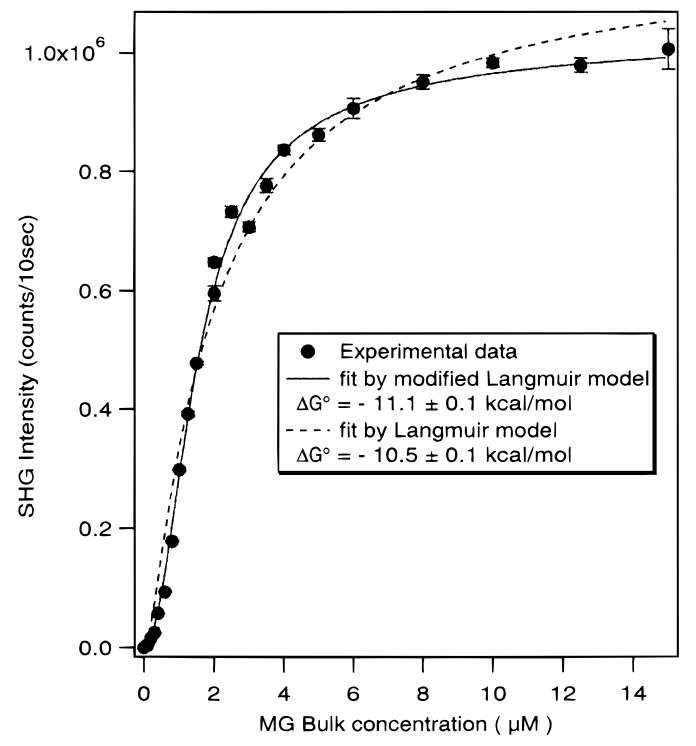

Figure 2. Malachite green (MG) adsorption isotherm on polystyrene sulfate (PSS) particle surface in aqueous solution. The modified Langmuir model differs from the Langmuir model by including the depletion of bulk solute molecules due to their adsorption to the microparticle surface.

microspheres (Polysciences). The particle surfaces are negatively charged owing to the presence of sulfate $\left(-\mathrm{SO}_{4}{ }^{-}\right)$groups. All samples were prepared using doubly distilled water. The solution $\mathrm{pH}$ was $4.30 \pm 0.04$.

The oil/water emulsion was prepared by mixing a $5 \mathrm{mM}$ 1-dodecanol (Aldrich, >99\%)/n-tetradecane (Aldrich, > 99\%) solution with a $5 \mathrm{mM}$ sodium dodecyl sulfate, SDS (Sigma, > 99\%)/water solution at a volume/volume ratio 1:9. 1-Dodecanol acts as a coemulsifier in the emulsion. The UV/vis turbidity measurements showed that the emulsions were stable for at least 2 days. The malachite green chloride (MG) was obtained from Aldrich Chemicals and used without further purification. The purity of MG was checked using high-pressure liquid chromatography (HPLC). All measurements were performed at $22^{\circ} \mathrm{C}$.

\section{Results and Discussions}

a. Adsorption of Molecules to Aqueous/Polymer Bead Interface: The Modified Langmuir Model. The adsorption isotherm of MG in an aqueous solution containing $4 \times 10^{8} \mathrm{PSS} /$ $\mathrm{cm}^{3}$ is shown in Figure 2. The Langmuir model of surface adsorption, modified to include the decrease in concentration of the species in the bulk solution due to adsorption to the microparticle interface, yields an excellent fit to the dependence of the SHG signal on the bulk adsorbate concentration. The Langmuir model ${ }^{23}$ assumes that the surface can be treated as a 
lattice of noninteracting sites with the adsorption process described as the "reaction" between bulk molecules (M) and empty surface sites (ES) to give filled sites (FS)

$$
\mathrm{M}+\mathrm{ES} \underset{k_{-1}}{\stackrel{k_{1}}{\rightleftharpoons}} \mathrm{FS}
$$

The kinetic equation is

$$
\frac{\mathrm{d} N}{\mathrm{~d} t}=k_{1} \frac{(C-N)}{55.5}\left(N_{\max }-N\right)-k_{-1} N
$$

where $N$ is the number of adsorbed molecules at the interface per unit volume, $N_{\max }$ is the maximum number of sites per unit volume, and $k_{1}$ and $k_{-1}$ are the rate constants for adsorption and desorption, respectively. $C$ is the total number of adsorbed and bulk molecules per unit volume. $(C-\mathrm{N}) / 55.5$ is the mole fraction of the adsorbing molecules in the bulk solution, where 55.5 is the molarity of water.

If the depletion of the bulk population due to adsorption to the interface is neglected, one obtains the usual expression for the Langmuir model

$$
\frac{\mathrm{d} N}{\mathrm{~d} t}=k_{1} \frac{(C-N)}{55.5}\left(N_{\max }-N\right)-k_{-1} N
$$

Equation 3 is called the Langmuir model (LM), and we refer to eq 2 as the modified Langmuir model (MLM).

The Langmuir model has been typically used to describe adsorption to flat interfaces. For such surfaces, the maximum surface site concentration $N_{\max }$ for a given adsorbate is usually much smaller than the bulk concentration $C$ of the adsorbing molecules. For this circumstance $N$ can be neglected in the $C$ $-N$ term appearing in eq 2. Unlike flat surfaces, the total surface area of a suspension of microparticles such as beads and emulsions can be very large. Hence the $N$ can be comparable with $C$, and therefore the depletion of adsorbing molecules in the bulk solution phase due to adsorption to the interface cannot be neglected.

At equilibrium, the rate of adsorption and the rate of desorption are equal, $\mathrm{d} N / \mathrm{d} t=0$. This yields for the Langmuir model, expressed in eq 3 , the equilibrium relative surface coverage $N / N_{\max }$

$$
\frac{N}{N_{\max }}=\frac{1}{1+\frac{55.5}{K C}}
$$

where $K=k_{1} / k_{-1}$ is the equilibrium constant of the adsorption "reaction". Although the absolute number of $N$ and $N_{\max }$ cannot be directly obtained from experiment, the relative surface coverage $N / N_{\max }$ can be obtained. Therefore the equilibrium constant $K$ can be determined from the measured $N / N_{\max }$ and use of eq 4. Equivalently the experiment yields the adsorption free energy $\Delta G^{\circ}$ using the thermodynamic relation

$$
\Delta G^{\circ}=-R T \ln K
$$

From the previous discussion we see that the Langmuir model does not provide information about the absolute number of $N$ or $N_{\max }$, when depletion of the bulk concentration of the adsorbing species can be neglected. However, when depletion becomes significant, we find that $N$ and $N_{\max }$ can be obtained from the adsorption isotherm experiment. From eq 2 we obtain an expression for the density of adsorbed molecules $N$, in terms of the total concentration $C$, the maximum density of adsorbed molecules $N_{\max }$, and the equilibrium constant $K$.

$$
\begin{aligned}
& \frac{N}{N_{\max }}= \\
& \frac{\left(C+N_{\max }+55.5 / \mathrm{K}\right)-\sqrt{\left(C+N_{\max }+55.5 / \mathrm{K}\right)^{2}-4 C N_{\max }}}{2 N_{\max }}
\end{aligned}
$$

The second harmonic electric field is proportional to the density of adsorbed molecules. It can be expressed in a normalized way, which is proportional to $N / N_{\max }$ given by eq 6. Second harmonic intensity, which is proportional to the square of the relative surface coverage, $\left(N / N_{\max }\right)^{2}$, is measured as a function of the total solute concentration $C$. If the modified Langmuir model is a proper description of the solute adsorption, then the SHG intensity vs $C$ data can be fit to eq 6 with the two parameters $K$ and $N_{\max }$. We compare both the Langmuir model and the modified Langmuir model with our experimental results. As seen in Figure 2 the Langmuir model gives an approximate fit to the experimental results, whereas the modified Langmuir gives an excellent fit. Depletion of the bulk population of the solute because of adsorption to the microsphere surface is clearly important. This is directly shown by comparing the value of $N_{\max }$ with the value of $C$ at which saturation occurs. The $N_{\max }$ obtained is $(1.8 \pm 0.2) \times 10^{6}$ per PSS particle, which corresponds to a concentration of $1.2 \pm 0.2 \mu \mathrm{M}$. From Figure 2 we see that the adsorption reaches saturation before 2 $\mu \mathrm{M}$. Therefore, depletion of the bulk MG molecule due to adsorption cannot be neglected, and the modified Langmuir model is necessary to describe the adsorption of the solute. The fit with the modified Langmuir model gives an adsorption free energy for MG to the PSS/water interface of $\Delta G^{\circ}=-11.1 \pm$ $0.1 \mathrm{kcal} / \mathrm{mol}$, which indicates a strong driving force for the adsorption of MG to the PSS/water interface.

b. Adsorption of Molecules to Oil Droplet/Aqueous Interfaces in Emulsions. As with the adsorption to the solution/polymer bead interface just discussed, the adsorption of molecules to the oil droplet/aqueous interfaces in an oil/water emulsion is not readily measured by traditional techniques. It is possible to determine the adsorption of solute molecules to planar liquid/liquid interfaces, such as planar oil/water interfaces by traditional methods, such as the measurement of the liquid/ liquid interface tension. ${ }^{15,16}$ However, even for the planar oil/ water interfaces, a drawback of the interface tension method is that it cannot differentiate among different solutes adsorbed to the interface. It is therefore a method that is restricted, even for planar interfaces, to solutions containing a single solute species. With the nonlinear spectroscopic methods, we can differentiate among the interface species by tuning the incident light to the identifying resonance frequencies of the various interface species. The nonlinear method can be used therefore to determine the population of the various solutes in a multicomponent interface. At the wavelength of the incident light $(846 \mathrm{~nm})$, the MG molecules are two-photon resonant, which gives a resonance-enhanced $\mathrm{SH}$ signal. The other molecules of the oil/water emulsion are not resonant, and thus their contributions to the $\mathrm{SH}$ signal are negligible. For the present study we selected a system that has been widely used as a model emulsion system, ${ }^{24}$ namely, the oil in water emulsion with sodium dodecyl sulfate (SDS) as surfactant and 1-dodecanol as coemulsifier (Scheme 2).

The second harmonic measurements of the malachite green adsorption from the water phase, in which it is soluble, to the 


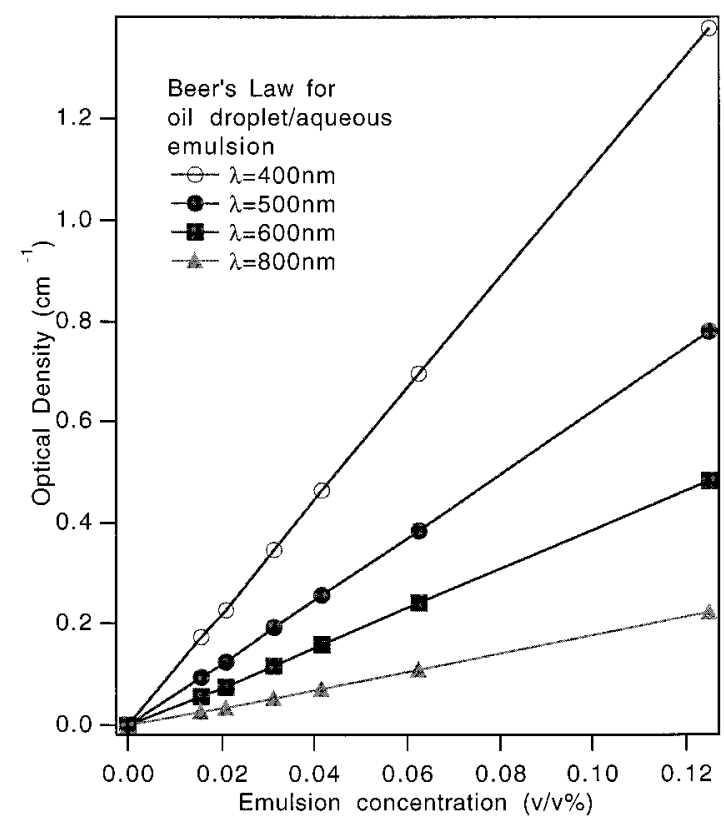

Figure 3. Turbidity measurement of oil droplet/aqueous emulsion. The turbidity at four different wavelengths show Beer's law behavior, indicating no size change of the emulsion droplets over a wide range of dilutions. The emulsion concentration is the volume fraction of the oil phase.

\section{SCHEME 2}

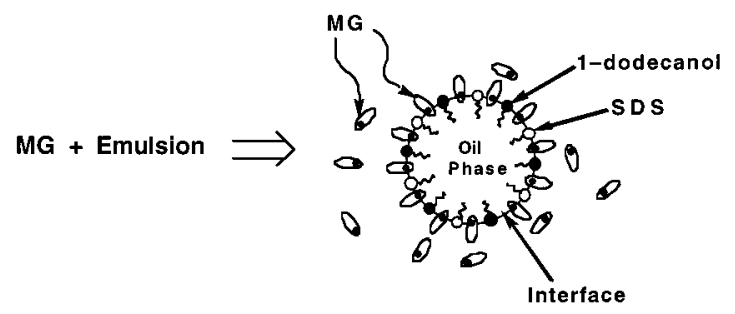

oil/water emulsion interfaces were carried out at two different emulsion densities $\left(5.2 \times 10^{10}\right.$ and $1.3 \times 10^{10}$ droplets $\left./ \mathrm{cm}^{3}\right)$, one being four times greater than the other. The different emulsion densities were achieved by diluting the initially prepared emulsion. To check that the emulsion is stable on dilution, the turbidity, i.e., the optical density, was measured at different dilutions and at different wavelengths. By stability it is meant that the average size and size distribution of oil droplets in the water phase remains the same upon dilution and their density undergoes a reduction equal to the volume dilution factor and does not decrease by a different amount. If dilution effected a change in the size distribution of oil droplets, then the turbidity would not change linearly with dilution. The results for turbidity measurements at different wavelengths, shown in Figure 3, show a linear dependence of the turbidity on dilution; i.e., the results are in agreement with Beer's law and thus show that the emulsion is stable on dilution. On applying Mie scattering theory to the turbidity data, we extracted the diameter of the oil droplet in an emulsion assuming a monodisperse distribution in droplet size. ${ }^{25,26}$ If the distribution in droplet sizes was very wide, i.e., polydisperse, then the diameters extracted from the turbidity data for different wavelengths would be different. This follows from the fact that the relative contributions of different size droplets to the scattering would not be the same for different wavelengths of the incident light. It was found for the tetradecane/water emulsions that the diameters obtained from the turbidity measurements were the same at the different wavelengths used $(350-800 \mathrm{~nm})$. This latter result indicates

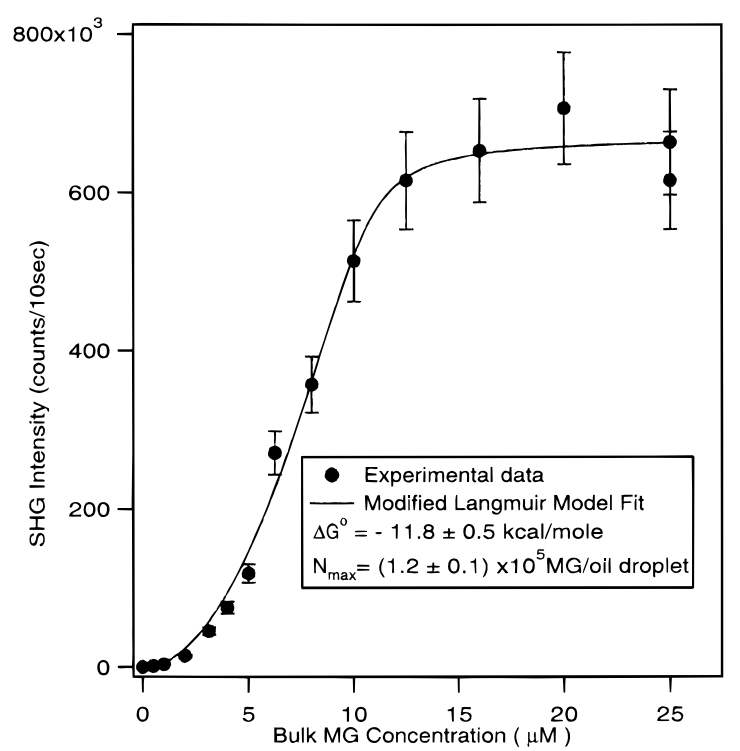

Figure 4. Adsorption isotherm of malachite green $(\mathrm{MG})$ to the oil droplet/water interfaces fitted to the modified Langmuir model. The droplet density was $5.2 \times 10^{10}$ droplets $/ \mathrm{cm}^{3}$.

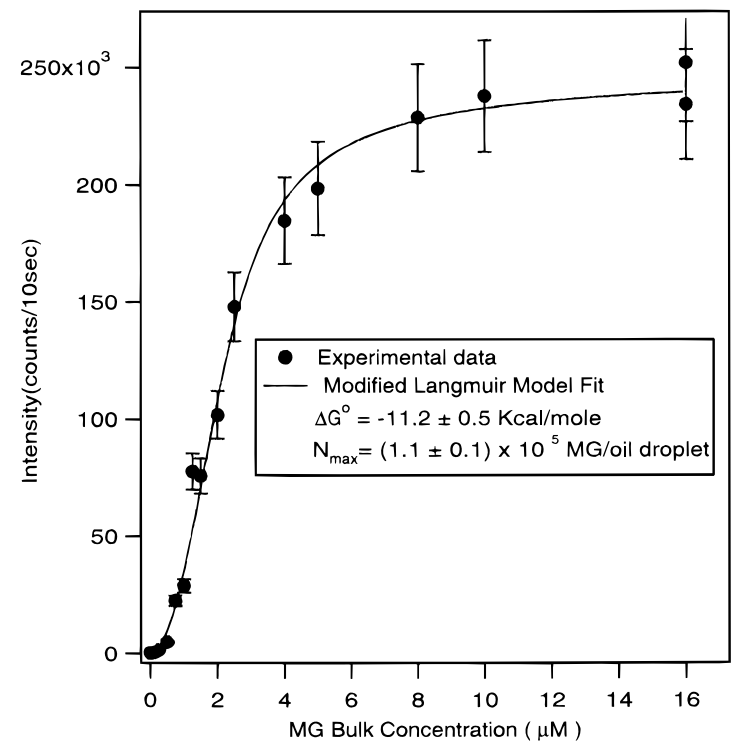

Figure 5. Adsorption isotherm of malachite green (MG) to the oil droplet/aqueous interfaces fitted by the modified Langmuir model. The droplet density is $1.3 \times 10^{10}$ droplets $/ \mathrm{cm}^{3}$ diluted by a factor of 4 from the emulsion shown in Figure 4.

that the distribution of droplet sizes is sharply distributed about an average diameter value of $230 \pm 3 \mathrm{~nm}$.

As with the polystyrene beads, the $\mathrm{SH}$ measurements of the MG adsorption as a function of the MG bulk concentration yielded the adsorption isotherm to the emulsion interfaces. Results of these measurements are shown in Figures 4 and 5 for two emulsions, which differed in the concentration (density) of oil droplets. The free energies of adsorption $\Delta G^{\circ}$ and the maximum density of interface sites $N_{\max }$ for the two emulsion densities were obtained from fitting the SH data to a modified Langmuir model for adsorption. At the higher droplet density, a value of $\Delta G^{\circ}=-11.8 \pm 0.5 \mathrm{kcal} / \mathrm{mol}$ and an $N_{\max }=10 \pm$ $0.8 \mu \mathrm{M}$ or equivalently $(1.2 \pm 0.1) \times 10^{5} \mathrm{MG}$ per oil droplet were obtained. For the lower density emulsion the corresponding results were $\Delta G^{\circ}=-11.2 \pm 0.5 \mathrm{kcal} / \mathrm{mol}$ and an $N_{\max }=$ $2.3 \pm 0.3 \mu \mathrm{M}$ or equivalently $(1.1 \pm 0.1) \times 10^{5} \mathrm{MG}$ per oil droplet. The same values within experimental error of $\Delta G^{\circ}$ 
obtained for the two different emulsions indicate that the oil droplet density is not affecting the adsorption of $\mathrm{MG}$ to the oil droplet/water interfaces in these emulsions.

The maximum number of adsorption sites per unit volume for $\mathrm{MG}$ is reduced by roughly a factor of 4 when the oil droplet concentration is reduced by a factor of 4 . In agreement with this it was found that the maximum number of $\mathrm{MG}$ adsorbed on each oil droplet remains the same, i.e., $(1.2 \pm 0.1) \times 10^{5}$ and $(1.1 \pm 0.1) \times 10^{5}$ sites per oil droplet for the higher and lower densities, respectively.

A comparison of the findings for $\Delta G^{\circ}$ for the adsorption of MG to the $n$-tetradecane/water emulsion interface with $\mathrm{MG}$ adsorption to the polystyrene sulfate bead/water interface shows that they are the same within our experimental precision. This result suggests that the interfaces, both of which consist of organic and water phases and negatively charged sulfate groups, are similar with respect to the MG adsorption process.

\section{Conclusion}

A second harmonic method is used to provide a new and promising way to investigate the interfaces of microscopic particles. In the present studies the adsorption free energies and the densities of malachite green at the aqueous/polystyrene sulfate bead interface and at the oil (tetradecane) droplet/aqueous interface in an oil/aqueous emulsion were obtained. The second harmonic measurements yield a free energy of adsorption to the aqueous interface of a $1.05 \mu \mathrm{m}$ diameter polystyrene bead, $\Delta G^{\circ}=-11.1 \pm 0.1 \mathrm{kcal} / \mathrm{mol}$, and a maximum number of adsorbate sites $N_{\max }=(1.8 \pm 0.2) \times 10^{6}$ per polystyrene bead. For the tetradecane droplets in water emulsion, the $\Delta G^{\circ}$ for adsorption is $-11.6 \pm 0.5 \mathrm{kcal} / \mathrm{mol}$ and an $N_{\max }=(1.2 \pm 0.1)$ $\times 10^{5}$ per droplet for a $230 \mathrm{~nm}$ diameter oil droplet emulsion. It is to be noted that the adsorption isotherm measurement on an oil/water emulsion surfaces is the first in situ adsorption isotherm measurement to our knowledge on microscopic liquid/ liquid particle interfaces. The use of this approach as a sensor method to quantitatively detect chemical and biochemical species without the need to introduce artificial tags is anticipated; the only requirement is that the surfaces have a second-order nonlinearity.

\section{References and Notes}

(1) Richmond, G.; Robinson, J. M.; Shannon, V. L. Prog. Surf. Sci. 1988, 28,1 .

(2) Shen, Y. R. Ann. Rev. Phys. Chem. 1989, 40, 327.

(3) Eisenthal, K. B. Аnnu. Rev. Phys. Chem. 1992, 43, 627.

(4) Corn, R. M.; Higgins, D. A. Chem. Rev. 1994, 94, 107.

(5) Eisenthal, K. B. Chem. Rev. 1996, 96, 1343.

(6) Giordmaine, J. A. Phys. Rev. A. 1963, 138, 1559

(7) Bloembergen, N. Nonlinear Optics; Wiley: New York, 1965.

(8) Terhune, R. W.; Maker, P. D.; Savage, C. M. Phys. Rev. Lett. 1963, 14,681

(9) Clays, D.; Persoons, A. Phys. Rev. Lett. 1991, 66, 2980.

(10) Song, Q.; Wan, C.; Johnson, C. D. J. Chem. Phys. 1994, 98, 1999.

(11) Akisipetrov, O. A.; Elyutin, P. V.; Nikultin, A. A.; Ostrovka, E. A. Phys. Rev. B 1995, 51, 17591.

(12) Wang, H.; Yan, E. C. Y.; Borguet, E.; Eisenthal, K. B. Chem. Phys. Lett. 1996, 259, 15.

(13) Vogel, V. Curr. Opin. Colloid Interface Sci. 1996, 1, 2.

(14) Wang, H.; Yan, E. C. Y.; Liu, Y.; Eisenthal, K. B. Unpublished results.

(15) Chattoraj, D. K.; Birdi, K. S. Adsorption and the Gibbs Surface Excess; Plenum Press: New York, 1984

(16) Ross, S. and Morrison, I. D. Colloidal Systems and Interfaces; John Wiley \& Sons: 1988 .

(17) Adamson, A. W. Physical Chemistry of Surfaces, 5th ed.; John Wiley \& Sons: New York, 1990.

(18) Myers, D. Surfaces, Interfaces, and Colloids-Principles and Applications; VCH Publishers: New York, 1990.

(19) Masel, R. I. Principles of Adsorption and Reaction on Solid Surface; John Wiley \& Sons: New York, 1996.

(20) Kipling, J. J. Adsorption from Solution of Nonelectrolytes; Academic Press: New York, 1965.

(21) Giles, C. H.; Nakhwa, S. N. J. Appl. Chem. 1962, 12, 266.

(22) Giles, C. H.; D'Silva, A. P.; Trivedi, A. S. Proc. Int. Symp. 1970, 1969,317

(23) Langmuir, I. J. Am. Chem. Soc. 1981, 40, 1361.

(24) Encyclopedia of Emulsion Technology; Becher, P., Ed.; Marcel Dekker: New York, 1983.

(25) Kerker, M. Electromagnetic Scattering Proceedings; Macmillan: New York, 1963; pp 122-131.

(26) Tabibian, R.; Heller, W.; Epel, J. N. J. Colloid Sci. 1956, 11, 195. 